\title{
One Action System or Two? Evidence for Common Central Preparatory Mechanisms in Voluntary and Stimulus-Driven Actions
}

\author{
Gethin Hughes, ${ }^{1,2}$ Simone Schütz-Bosbach, ${ }^{3}$ and Florian Waszak ${ }^{1,2}$ \\ ${ }^{1}$ Université Paris Descartes, Sorbonne Paris Cité, 75270 Paris, France, ${ }^{2}$ CNRS, Laboratoire Psychologie de la Perception, UMR 8158, 75006 Paris, France, \\ and ${ }^{3}$ Max Planck Institute for Human Cognitive and Brain Sciences, 04103 Leipzig, Germany
}

Human behavior is comprised of an interaction between intentionally driven actions and reactions to changes in the environment. Existing data are equivocal concerning the question of whether these two action systems are independent, involve different brain regions, or overlap. To address this question we investigated whether the degree to which the voluntary action system is activated at the time of stimulus onset predicts reaction times to external stimuli. We recorded event-related potentials while participants prepared and executed left- or right-hand voluntary actions, which were occasionally interrupted by a stimulus requiring either a left- or right-hand response. In trials where participants successfully performed the stimulus-driven response, increased voluntary motor preparation was associated with faster responses on congruent trials (where participants were preparing a voluntary action with the same hand that was then required by the target stimulus), and slower responses on incongruent trials. This suggests that early hand-specific activity in medial frontal cortex for voluntary action trials can be used by the stimulus-driven system to speed responding. This finding questions the clear distinction between voluntary and stimulus-driven action systems.

\section{Introduction}

Human behavior is often classified into two classes of actions, reactions to external events and voluntary actions (Haggard, 2008; Passingham et al., 2010), thought to be controlled by different brain systems (Krieghoff et al., 2011). Studies using both event-related potentials (ERPs) (e.g., Waszak et al., 2005) and functional imaging (Deiber et al., 1999; Jenkins et al., 2000) suggest that while voluntary actions involve the fronto-median cortex, stimulus-driven actions are controlled via parietal and premotor cortices (Haggard, 2008). However, monkey studies show that supplementary motor area (SMA) neurons discharge to both self-initiated and externally cued movements, indicating that this region is involved in both types of action (Romo and Schultz, 1987; Kurata and Wise, 1988).

Importantly, imaging techniques and single-cell recordings alone may be insufficient to determine the relationship between voluntary and stimulus-driven motor preparation. A given area may be composed of different subparts, each being involved in one type of action. Alternatively, an area, or even a particular cell, might be involved in both action types but fulfill different func-

\footnotetext{
Received May 5, 2011; revised Aug. 30, 2011; accepted 0ct. 3, 2011.

Author contributions:G.H., S.S.-B., and F.W. designed research; G.H. performed research;G.H. and F.W. analyzed data; G.H., S.S.-B., and F.W. wrote the paper.

This work was supported by collaborative research grants from the Agence Nationale de la Recherche and the Deutsche Forschungsgemeinschaft (ANR-08-FASHS-13 and Schu2471/1-1). We are grateful to Lea Friedrich and Lukasz Grzeczkowski for assistance with data collection.

Correspondence should be addressed to Gethin Hughes, Laboratoire Psychologie de la Perception, Université Paris Descartes, 45 Rue des Sts Pères, 75270 Paris Cedex 06, France. E-mail: hughes.gethin@gmail.com.

DOI:10.1523/JNEUROSCI.2256-11.2011

Copyright $\odot 2011$ the authors $\quad 0270-6474 / 11 / 3116692-08 \$ 15.00 / 0$
}

tions. Moreover, the increased complexity associated with voluntary actions confounds any observed differences (Nachev et al., 2008).

We believe that the only way to truly determine the relationship between voluntary and stimulus-driven actions is to test whether activity in one system is transferable to the other. We did so by investigating the performance of the stimulus-driven action system [measured by reaction times (RTs)] as a function of the state of the voluntary action system [measured by readiness potential (RP) amplitude]. Participants prepared left- or right-hand voluntary actions that could be interrupted by a stimulus requiring either a left- or right-hand response, making the response either congruent or incongruent with concurrent voluntary action preparation. We tested whether the amplitude of the RP at stimulus onset predicted participants' response time to the stimulus. As discussed below, a similar behavioral task has previously been used by Obhi et al. (2009a,b).

We tested our results against four hypotheses. First, no association between prestimulus activity and RTs would suggest independence of the two systems. Second, previous behavioral studies (e.g., Obhi and Haggard, 2004) show that stimulus-driven responding is slowed during concurrent voluntary action preparation, suggesting that not only are the two systems independent but that inhibitory mechanisms may exist between them. This conclusion is consistent with observations from patients with utilization behavior (e.g., Boccardi et al., 2002) who show an inability to refrain from stimulus-driven actions following SMA lesions. According to this account, larger prestimulus RPs would be associated with slower stimulus-driven responses for both congruent and incongruent trials. Alternatively, any prestimulus 

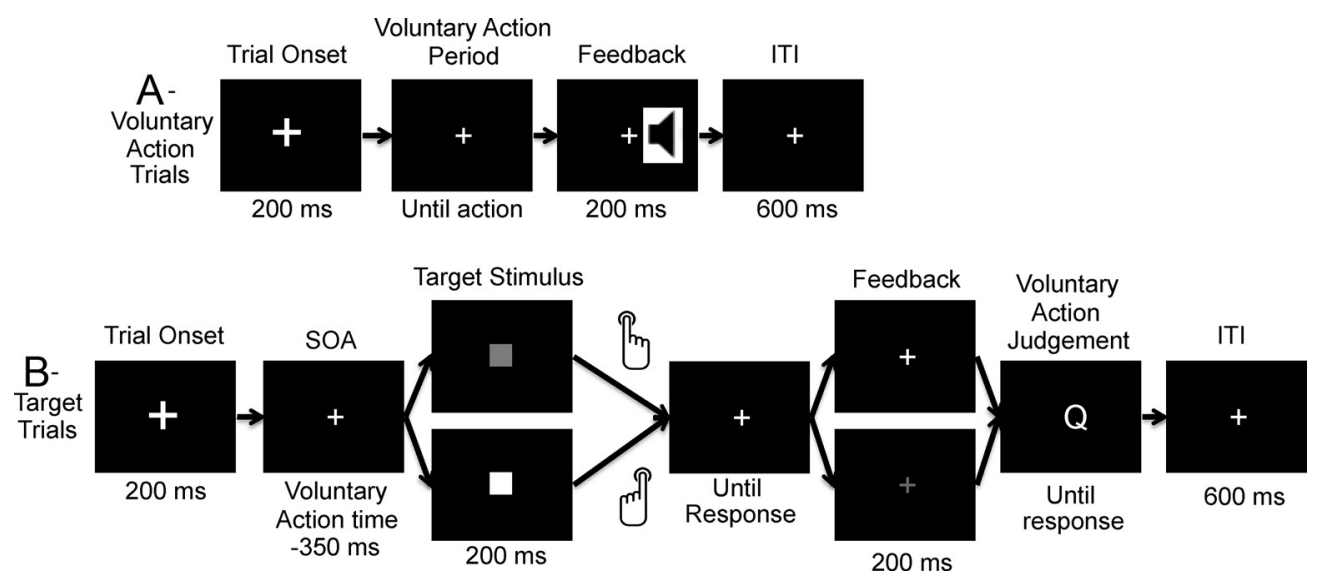

Figure 1. Trial sequence for voluntary action trials $(\boldsymbol{A})$ and target trials $(\boldsymbol{B})$. ITI, Intertrial interval; SOA, stimulus onset asynchrony.

negativity might reflect general anticipatory processes as indexed by the contingent negative variation (Walter et al., 1964), leading to smaller RTs for congruent and incongruent trials. Finally, the voluntary and stimulus-driven systems might overlap to such a degree that the preparation of the voluntary action is transferable to stimulus-driven preparation. If so, RTs should be faster when voluntary action preparation is more advanced for congruent trials only, with the opposite pattern for incongruent trials.

\section{Materials and Methods}

Participants. Data were collected from 28 paid volunteers. All participants were right handed, had normal or corrected-to-normal vision, and had no history of epilepsy. Individuals who were taking psychoactive drugs were excluded from participating. Six participants were excluded from the analysis, as they were unable to perform the task correctly. These participants always rated that their planned voluntary action was consistent with the direction required by the imperative stimulus (see below for details of this rating). Given that these stimuli were not predictable, this likely reflects a response bias to simply press the same button for the response and for the question regarding their voluntary action. Four additional participants were excluded from the analysis described below because of technical problems with the EEG recording, leaving 10 female and 8 male participants, with a mean age of 25 years and 1 month (range, 21-31 years). All participants signed an informed consent before the experimental session and were free to withdraw at any point.

Experimental procedure. The experiment began with a practice phase where participants were introduced to the task in stages. The first practice task was constructed of 80 trials where either a blue or a red square was presented in the center of the screen on each trial (40 of each, randomly ordered). Participants were required to make a speeded response to the squares with a right or left key press, with the stimulus-response mapping counterbalanced across participants. This task also served as a baseline to record participants mean response times to the target stimuli. In the second practice task, participants were required to perform voluntary key presses around $1.5 \mathrm{~s}$ after the start of each trial, as defined by a $200 \mathrm{~ms}$ enlargement of the fixation cross. Participants were instructed that they could respond with either the left hand (L) or the right hand (R) and that they should not act in a systematic manner (e.g., L-R-L-R). Instead they were informed to decide on each trial which button they wanted to press. They were also informed that they should respond with each hand approximately equally. Participants received auditory feedback with regard to the timing of their voluntary actions. One tone signified that they had responded too quickly (under $1200 \mathrm{~ms}$ ) and a second tone signified slow responses (over $2000 \mathrm{~ms}$ ). This training consisted of 80 trials.

The final task in the practice phase was the experimental task (see Fig. 1). This involved combining the two previous tasks, such that participants were required to make voluntary key presses around $1.5 \mathrm{~s}$ after trial onset (voluntary action trials; Fig. $1 \mathrm{~A}$ ). However, on some trials a blue or red square would appear that would then require them to make the appropriate stimulus-driven response (target trials; Fig. $1 B$ ). Each block consisted of 84 trials, of which 54 were voluntary action trials (Fig. $1 A$ ) and 30 were trials where a target stimulus appeared, henceforth referred to as target trials (Fig. $1 \mathrm{~B}$ ). Target trials were randomly ordered with the exceptions that they did not occur in the first four trials of each block and that there were never more than two consecutive target trials. This random ordering of target trials ensured that up to the point of target presentation participants would not know that a stimulus would appear, and therefore voluntary action preparation at the point of stimulus presentation should be similar to the period before a voluntary action. The timing of the target stimulus was determined relative to the onset of the action for voluntary action trials, such that it appeared $350 \mathrm{~ms}$ before the mean onset of the action for the last 20 voluntary action trials. This time interval was chosen so that participants should already have begun preparing a voluntary action on these trials, but they should not be in the very late stages of action preparation. However, given that the voluntary action time would also vary from trial to trial, we would likely have trials where action preparation was more advanced and trials where action preparation was less advanced. Indeed on some trials, participants made their voluntary actions before the onset of the target stimulus. On these trials the target stimulus was no longer presented. This occurred on an average of $6.6 \%$ of all trials where a target stimulus would have been presented, resulting in a mean of fewer than two cancelled target trials per block. This natural variability should allow us to investigate the association between prestimulus voluntary action preparation and stimulusdriven responding. In addition, the use of a moving average to calculate the onset of the target stimulus should discourage participants from waiting to see whether the stimulus would appear before programming their action, since as their voluntary action times became slower, the target stimulus would begin to appear later.

Following the response to the target, a white fixation (correct response) or red fixation (incorrect response) acted as feedback to the participants. Following this feedback, participants were required to rate their voluntary action preparation by answering the following question: "Before the square appeared, which button were you planning to press?" They responded with one of four buttons on the response pad with the following options (from left to right): certain left, maybe left, maybe right, and certain right. The certain options were to be used when participants had confidence in their judgment, while the middle two categories were to be used if they were not completely sure which button they had been planning to press.

Following the practice phase, participants were fitted with the EEG recording caps. Next they completed a short block of the stimulusresponse training (20 trials), followed by one block of the voluntary actions (80 trials), to reestablish their response rate. Following these practice tasks they completed 16 blocks of the experimental task. Participants continued to receive feedback during the experimental task with respect to both the accuracy of the target response (visual feedback) and their voluntary action times (auditory feedback). In addition, at the end 
of each block participants were informed about their mean accuracy for the target stimulus, as well as the number of voluntary actions in the correct time window and the number of voluntary actions produced with each hand.

EEG recording and data preprocessing. EEG data were recorded from 63 scalp locations using an EGI system (Electrical Geodesic Inc.). The EEG was digitized at $500 \mathrm{~Hz}$ with a $0.01-200 \mathrm{~Hz}$ bandpass filter referenced to the vertex. EEG analysis was conducted using EEGLAB (Delorme and Makeig, 2004) and custom-built Matlab scripts. The data were resampled offline to a $250 \mathrm{~Hz}$ sample rate, with a notch filter from 45 to $55 \mathrm{~Hz}$ to remove line noise. Epochs were generated from -3000 to $1000 \mathrm{~ms}$ relative to stimulus onset, with a $100 \mathrm{~ms}$ prestimulus baseline correction for target trials and a $100 \mathrm{~ms}$ pre-action baseline correction for voluntary action trials. Initial artifact rejection was conducted in a semiautomatic manner (in EEGLAB) by rejecting epochs with activity above 100 or below $-100 \mu \mathrm{v}$, as well as rejecting trials where activity at any time point for any electrode was more that 5 standard deviations from the mean activity for that epoch. Any channels that contributed to the rejection of many epochs were considered for removal and later interpolation. Frontal channels that showed large amplitude blink activity were also excluded from this first pass of semiautomatic artifact rejection. Ocular artifact correction was conducted in EEGLAB in Matlab using independent component analysis (Delorme and Makeig, 2004). Following removal of eye blinks and eye movements, noisy channels were replaced by an interpolated weighted average from surrounding electrodes. Data were then re-referenced to the common average. A final round of semiautomatic artifact rejection with a threshold of $\pm 80 \mu \mathrm{v}$ was used to remove any remaining artifacts.

To assess prestimulus motor activity, we re-baselined our ERPs to the $100 \mathrm{~ms}$ period before the onset of the trial (the large fixation cross). RP was measured at electrode $\mathrm{Cz}$ (electrode 63 on EGI sensor net), with LRP calculated in line with Coles (1989) using electrodes FC3 (electrode 17) and FC4 (electrode 54). Analysis on the association between prestimulus $\mathrm{RP} / \mathrm{LRP}$ amplitude and RT were calculated by taking the average amplitude in a $100 \mathrm{~ms}$ time window before target onset. Since LRP is normally calculated by taking the average of left- and right-hand responses, we used a single subtraction for each trial to measure prestimulus motor lateralization by calculating the activity over the hemisphere contralateral to the response hand minus ipsilateral to the response hand. We used these values to calculate a median split of the RP/LRP amplitude and extracted mean RTs for the trials either side of the median RP/LRP. All ERPs are presented with a low-pass filter of $20 \mathrm{~Hz}$ for visual presentation purposes.

\section{Results}

\section{Behavioral results}

Voluntary action trials

Mean average voluntary action time was 1529 ms, with a mean standard deviation of $300 \mathrm{~ms}$. Participants made on average $83 \%$ of their responses within the required time window, with $10.4 \%$ of their responses occurring faster than $1200 \mathrm{~ms}$, and the remaining $6.3 \%$ occurring later than $2000 \mathrm{~ms}$. This suggests that participants were generally able to time their voluntary actions to occur within the required time period but that there was also some trial-to-trial variation in the time of their action. This variation is important to ensure that the target stimulus could appear at varying levels of voluntary action preparation.

\section{Ratings of voluntary action for target trials}

This analysis focuses on how participants responded to the question regarding their prepared voluntary action on target trials. Following their response to the target, participants were asked to rate, using a four-point scale (certain left, maybe left, maybe right, certain right), which action they had been preparing before the onset of the stimulus. Participants were significantly more likely to rate that they were sure of their response ( $82.3 \%$ of trials) than they were to say that they had maybe prepared left or right
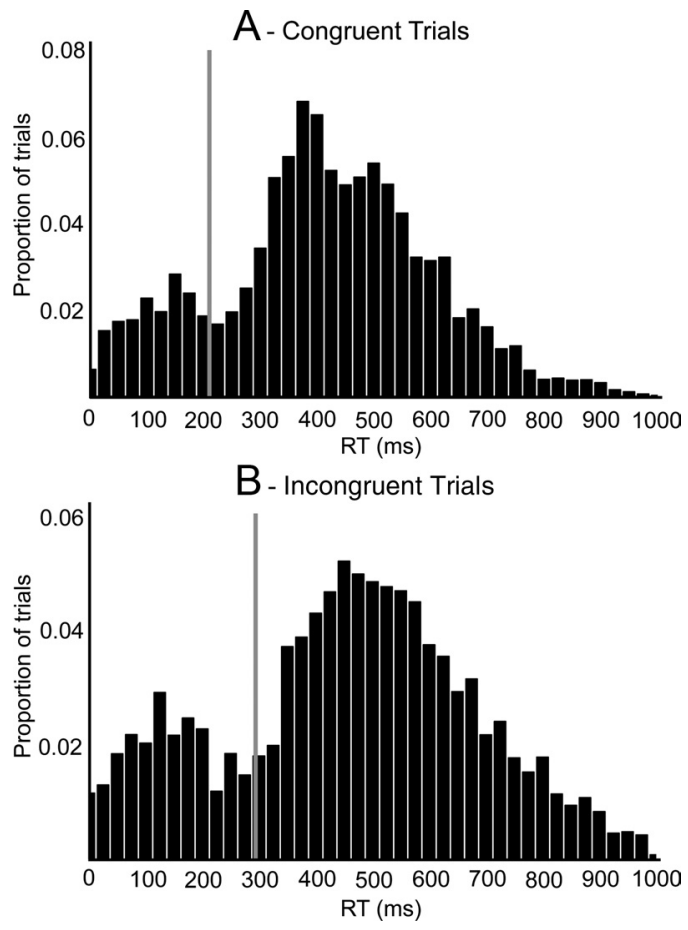

Figure 2. RT distributions in 25 ms time windows from 0 to 1000 ms for congruent $(\boldsymbol{A})$, and incongruent $(\boldsymbol{B})$ responses averaged across all participants. Values on the $y$-axis correspond to the proportion of trials falling in each RT bin, with the $x$-axis representing RT. The vertical line represents the mean RT cutoff for separating the two distributions.

$\left(17.7 \%\right.$ of trials; $\left.t_{(17)}=5.83 ; p<0.001\right)$. Participants appeared somewhat more inclined to say that the target stimulus required the same hand response that they were already preparing $(43.4 \%$ of trials) rather than the opposite hand (39\% of trials). However this difference was not significant $\left(t_{(17)}=1.75 ; p=0.1\right)$. This suggests that participants were able to rate their voluntary action preparation, and on the majority of trials they felt confident about this judgment. Given the small number of responses in the middle conditions (maybe left and maybe right), subsequent analyses were conducted collapsing across the maybe and certain conditions for responses that were congruent with the prepared voluntary action (i.e., those trials where participants reported preparing the same hand action as that required by the target stimulus) and those that were incongruent. These will henceforth be referred to as congruent and incongruent trials. Where possible, behavioral responses from the middle two categories were also analyzed using a subset of participants $(N=9)$ who had at least 10 responses in each of these middle categories. Analysis of ERPs was confined to congruent and incongruent trials (i.e., collapsed across certain and maybe voluntary action ratings), since only three participants had sufficient responses in the middle two categories $(>20)$ to produce reliable ERPS.

\section{Responses on target trials}

We first plotted the RT distributions for each participant to investigate the possibility that some responses after the onset of the target stimulus represented the execution of the voluntary action rather than a response to the stimulus. Figure 2 shows the RT distributions for all trials (correct and incorrect) averaged across all participants for congruent and incongruent trials. The vast majority of participants showed a strong bimodal distribution probably reflecting the separation of target responses (with slower RTs) and the execution of the planned voluntary action 
(fast RTs). This bimodality is clearly reflected in the average RT histograms in Figure 2, $A$ and $B$. The vertical line represents the mean RT cutoff, which we used to separate these distributions for each participant. The mean cutoff was $210 \mathrm{~ms}$ for congruent trials and $294 \mathrm{~ms}$ for incongruent trials. Analysis of accuracy for these groups of trials revealed a significant interaction between distribution (fast vs slow RTs) and congruency $\left(F_{(1,17)}=956, p<\right.$ 0.001). Accuracy for trials above the cutoff was significantly greater for congruent trials (mean accuracy $=0.97$ ) as compared to incongruent trials (mean accuracy $=0.94 ; t_{(17)}=2.9, p<$ $0.05)$. However, this difference was much more pronounced for the fast RT trials (mean accuracy congruent $=0.87$; mean accuracy incongruent $=0.0062 ; t_{(17)}=46.2, p<0.001$ ). This finding is consistent with the suggestion that trials below the cutoff were predominantly those where participants performed their voluntary action rather than responding to the target stimulus, since when their voluntary actions were congruent with the target stimulus, the response would be classed as correct. For example, if participants were preparing a left-hand voluntary action when a stimulus appeared requiring a left-hand response (a congruent trial), execution of the voluntary action (a left-hand button press) would result in a button press classed as correct with respect to the target. In contrast those voluntary actions that were executed on incongruent trials would always lead to an incorrect response, as highlighted by the fact that almost none of the fast responses to incongruent trials were correct. In subsequent analysis we divided the data on target trials into "voluntary actions on target trials," which are below the RT cutoff, and genuine "target responses," which are above the RT cutoff. As described above, this distinction is justified by the significantly reduced accuracy for the fast responses on incongruent trials.

This clear separation between the RT distributions for executed voluntary actions on target trials and target responses points to the possibility that participants first check their voluntary action preparation and then evaluate the stimulus before continuing to perform a stimulus-driven action. A number of previous studies (Obhi and Haggard, 2004; Astor-Jack and Haggard, 2005; Obhi et al., 2009a, 2009b) have shown that RTs in blocks with concurrent voluntary actions were significantly longer than responses to the same stimuli in blocks where voluntary actions were not being prepared. Indeed, in the current experiment RTs in the practice block where participants were only required to respond to the colored squares were significantly faster (mean RT $=350.4 \mathrm{~ms}$ ) than RTs for congruent trials in the experimental blocks (mean RT $=474.2 \mathrm{~ms}$ ) where participants were also required to perform voluntary actions $\left(t_{(17)}=6.3, p<\right.$ $0.001)$. This was the case even including those very fast responses (mean RT $=410.5 \mathrm{~ms}$ ), which were in fact likely not truly stimulus driven $\left(t_{(17)}=3.9, p<0.01\right)$. These findings are therefore consistent with previous studies that suggest that preparation of voluntary actions impedes speeded responding to an external stimulus. However, despite an overall cost in RT observed when switching from a voluntary action to a stimulus-driven action, this does not rule out the possibility that voluntary action preparation can be facilitate stimulus-driven responding (as highlighted in the analysis below). We will return to the possible mechanisms of the overall RT cost in the general discussion section.

Our subsequent analysis investigated whether the degree to which participants had prepared their voluntary action influences the amount of interference in target responding. As outlined in the introduction, this was to assess whether activation of the voluntary action system could be used to improve perfor- mance of the stimulus-driven system. We first investigated this behaviorally by using RTs dependent on participants' ratings of their voluntary action preparation. This analysis was conducted using only those trials above the RT cutoff, as these exclude the trials where participants actually performed their voluntary action. A similar filtering of responses was conducted in previous studies (Astor-Jack and Haggard, 2005; Obhi and Haggard, 2004; Obhi et al., 2009a, 2009b) where all trials with RTs under $100 \mathrm{~ms}$ were removed. The higher RT cutoff in our study reflects the fact that participants were performing a choice RT task rather than a simple RT task.

Analysis of mean correct RTs revealed that responses were significantly faster for congruent trials $(476.5 \mathrm{~ms})$ as compared to incongruent trials $\left(559.9 \mathrm{~ms} ;\left(t_{(17)}=6.67, p<0.001\right)\right.$. We also found that in the subset of participants who used the middle two classifications of their voluntary action, RTs were significantly faster for the completely congruent trials $(457.3 \mathrm{~ms})$ compared to the partially congruent trials $\left(540.5 \mathrm{~ms} ; t_{(7)}=7.3, p<0.001\right)$. For incongruent trials, this comparison ( $595.8 \mathrm{~ms}$ for partially incongruent vs $637.7 \mathrm{~ms}$ for incongruent) was not significant $\left(t_{(7)}=\right.$ $1.77, p>0.1)$. The direction of these results points to the possibility that more advanced preparation of a voluntary action may aid responding to an external stimulus that is congruent with the intended action, providing initial evidence of a possible transfer of voluntary action preparation to stimulus-driven actions.

Overall, the pattern of behavioral results suggests that participants were generally able to respond correctly to the target stimulus despite having already prepared a voluntary action, which on many occasions was with the opposite hand to that required by the stimulus. By separating the RT distributions based on their bimodality, we were able to isolate those trials where participants genuinely responded to the target stimulus. The congruency of participants' voluntary action preparation with the response required by the target stimulus strongly influenced both their accuracy and their response time to the target stimulus, such that they were faster to respond and more accurate on congruent trials. In addition, participants' own ratings of their awareness of the laterality of their intended response suggests that more advanced voluntary action preparation can in fact aid speeded responding.

\section{ERP results}

\section{Motor preparation}

To investigate the relationship between voluntary action preparation and responses to the target stimulus, our ERP analysis primarily focused on the RP and LRP before the onset of the stimulus and how these affect RTs to target stimulus. Before discussing this analysis, we present, in Figure 3, the RP (Fig. 3A) and LRP (Fig. $3 B$ ) for voluntary actions on voluntary action trials. RP appeared to onset around $1 \mathrm{~s}$ before action onset. One-sample $t$ tests revealed that RP amplitude began to differ from 0 at -964 ms. LRP amplitude began to significantly differ from 0 at -580 $\mathrm{ms}$. These onset times are comparable to other studies where RP onsets from 1 to $2 \mathrm{~s}$ before action (Libet et al., 1983; Haggard and Eimer, 1999) and LRP onsets around $700 \mathrm{~ms}$ before action (Haggard and Eimer, 1999).

Figure $3 C$ shows the RP prior stimulus onset for target trials. RPs are plotted separately for trials where participants performed their planned voluntary action despite the presence of the target stimulus - as identified by the individual participant RT distribution-and for trials where participants genuinely responded to the target stimulus itself. For the voluntary actions on target trials, we included both "correct" and "incorrect" responses to 
highlight voluntary action preparation, since here participants did not respond to the stimulus but followed their voluntary preparation. For genuine target responses, we only included correct responses to assess the level of voluntary action preparation that could be successfully overridden by the requirement to respond to the stimulus. The RP (averaged across all conditions) was different to 0 from $664 \mathrm{~ms}$ before target onset. Since RP onset in the voluntary action condition was around 1000 ms before stimulus onset, the onset of this RP is consistent with the fact that the stimulus appeared, on average, $350 \mathrm{~ms}$ before the point at which participants were planning to perform their action. ANOVA in the $500 \mathrm{~ms}$ before stimulus onset revealed no significant effect of action type (voluntary actions vs genuine target responses, $F<1$ ) or congruency $(F<1)$ and no significant interaction $(F<1)$. This suggests that the size of the readiness potential at stimulus onset did not influence whether participants aborted their voluntary action and reprogrammed the stimulus-driven response, nor did it influence the rating of their voluntary action preparation.

Figure $3 D$ shows the LRP before target onset for congruent and incongruent trials separately for both genuine target responses as well as for trials where participants executed their voluntary actions. ANOVA in the $300 \mathrm{~ms}$ before stimulus onset revealed a significant main effect of congruency $\left(F_{(1,17)}=18.8, p<0.001\right)$ as well as a significant congruency by action type (voluntary action execution versus genuine target responses) interaction $\left(F_{(1,17)}=10.8, p<0.01\right)$. This interaction was driven by the presence of a significant effect of congruency for trials where participants performed their voluntary actions $\left(t_{(17)}=4.5, p<0.001\right)$, but not for genuine target responses $\left(t_{(17)}=1.1\right)$. This shows that for those trials where participants' hand-specific action preparation had reached an advanced stage, they were no longer able to overcome their voluntary action to perform the stimulus-driven response. This finding further validates the division of trials based on their bimodality and also shows that participants were genuinely preparing voluntary actions to be executed around the time that the stimulus appeared. It is also important to note that the action occurred several hundred milliseconds earlier for the voluntary actions on target trials than for the target responses themselves. As such, this closer proximity to the action onset is reflected in greater LRPs for these trials. The scalp maps, separated for leftand right-hand responses (Fig. $3 E-J$ ), show a strong central topography for the target responses (Fig. 3G,J), highlighting the lack of significant lateralized motor activity for these trials. In contrast, both the voluntary actions on voluntary action trials target responses $(\mathbf{G}, \mathbf{J})$.

\section{Readiness Potential Lateralized Readiness Potential}

A

B

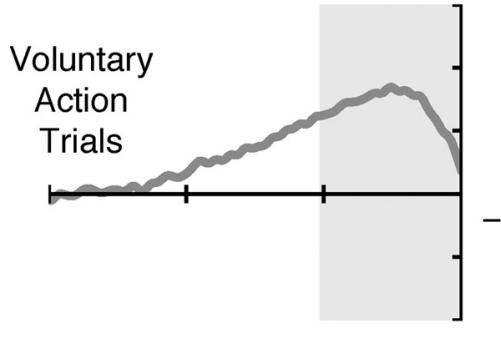

C

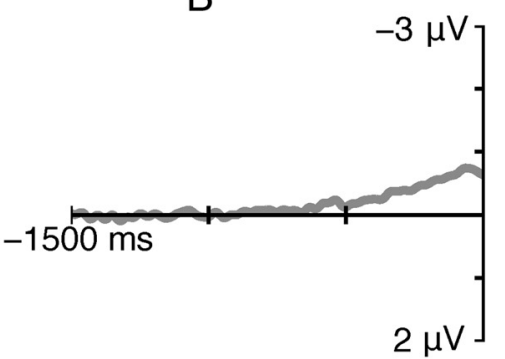

C

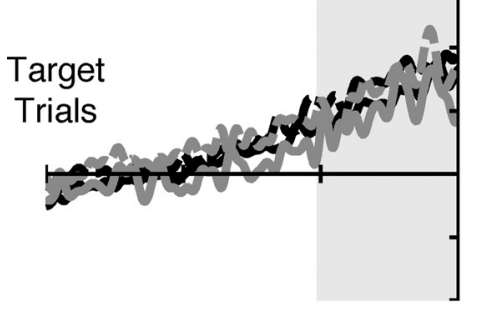

D

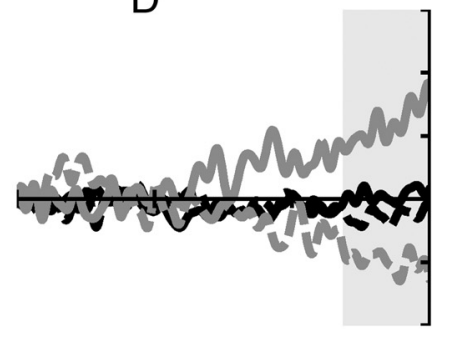

Voluntary Actions Target Responses
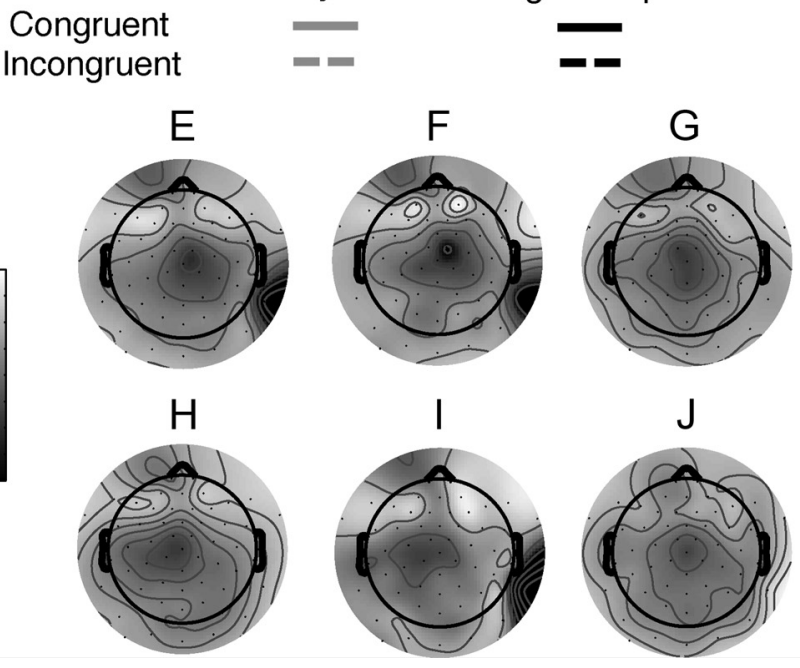

Figure 3. Motor activation before action onset for voluntary action trials $(A, B)$ and stimulus onset for target trials $(C, D)$. $A, B$, $\operatorname{RP}(\boldsymbol{A})$ and $\operatorname{LRP}(\boldsymbol{B})$ onset before action onset for voluntary action trials. $\boldsymbol{C}, \boldsymbol{D}, \mathrm{RP}(\boldsymbol{C})$ and $\operatorname{LRP}(\boldsymbol{D})$ before $S 2$ onset $(0 \mathrm{~ms})$ for target and black lines indicate trials where participants genuinely responded to the target stimulus. Solid lines indicate trials where the S2 stimulus was congruent with the intended voluntary action, and dashed lines indicate incongruent trials. LRP plotted relative to the correct response to the target stimulus, such that negative values correspond to activation of the hand required by the target stimulus. $\boldsymbol{E}-\boldsymbol{J}$, Topographic plots show ERP amplitude averaged over the $500 \mathrm{~ms}$ period before action/stimulus onset for left-hand $(\boldsymbol{E}-\boldsymbol{G})$ and right-hand actions $(\boldsymbol{H}-\boldsymbol{J})$, for voluntary actions on voluntary action trials $(\boldsymbol{E}, \boldsymbol{H})$ and on target trials $(\boldsymbol{F}, \boldsymbol{I})$ as well as for

(Fig. $3 E, H$ ) and the voluntary actions on target trials (Fig. $3 F, I$ ) show a central and contralateral negativity. This lateralization is predominantly made up of a contralateral negativity, with little or no ipsilateral inhibition (positivity) as is often observed with choice LRPs for stimulus-driven tasks (Meynier et al., 2009). This could reflect a difference in the balance between contralateral activation and ipsilateral inhibition between voluntary and stimulus driven actions, or it might reflect a task-specific strategy to maintain the possibility to switch action at the last moment if required.

The presence of an LRP difference between voluntary actions on target trials and target responses despite no difference in the same comparison for the RP is perhaps somewhat counterintui- 

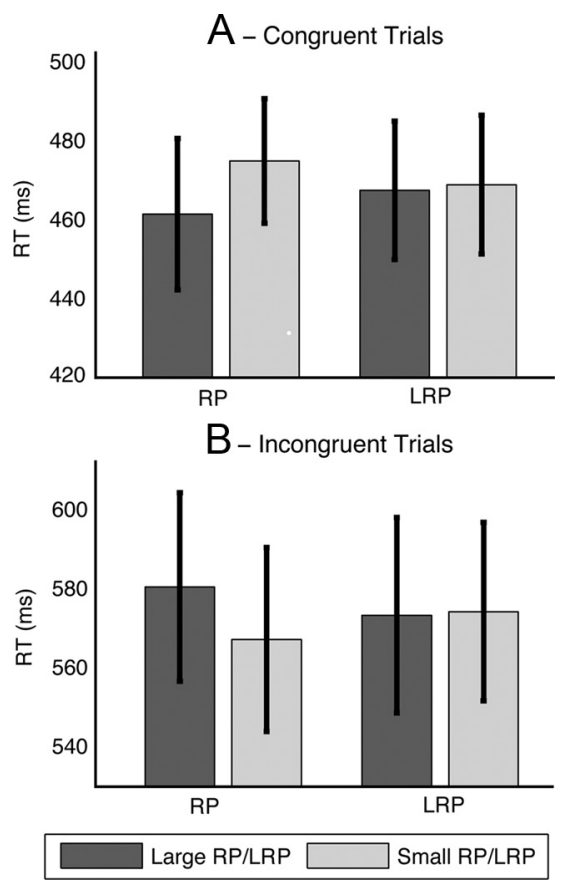

Figure 4. RT dependent on prestimulus RP and LRP for congruent $(\boldsymbol{A})$ and incongruent $(\boldsymbol{B})$ trials separately. Error bars show standard error.

tive, because if RP amplitude reflects motor preparation, how can the LRP be more advanced while RP suggests that motor preparation appears to be at the same stage? One possible explanation is that in some trials participants specified the response hand only at a very late stage of motor processing, while in other trials they specified this early in the trial. LRP differences have been previously reported in the absence of RP modulation before voluntary actions (Hughes and Waszak, 2011), suggesting that increased motor lateralization can occur in the absence of increased generalized motor preparation.

The absence of a significant LRP for the target responses also suggests that little or no M1 activation was present for those trials where participants made the stimulus-driven response. Despite the absence of any laterality, participants were able to rate the direction of their prepared voluntary response in a meaningful way (such that it has an effect of RTs and accuracy), suggesting that some information about the laterality of their response was present but perhaps had not yet been translated into an action command in primary motor cortex.

\section{Target responding dependent on motor readiness}

Our final analysis investigated the relationship between activation of the motor system at the time of stimulus onset and participants' response to the target stimulus. Our previous analysis of motor potentials showed that LRP and not RP amplitude at stimulus onset was associated with the ability of participants to successfully overcome their voluntary action. In this analysis we focus only on these genuine target responses to determine whether LRP or RP amplitude is associated with accuracy or reaction times to the target stimulus. Figure 4 shows the RT dependent on LRP and RP amplitude. ANOVA for RTs showed a significant three-way interaction between measure (RP/LRP), activation (large/small), and congruency, $F_{(1,17)}=6.12, p<0.05$. This three-way interaction was driven by a significant interaction between activation and congruency for $\operatorname{RP}\left(F_{(1,17)}=31.2, p<\right.$ $0.001)$, but not for $\operatorname{LRP}\left(F_{(1,17)}=1.28\right)$. For congruent trials a larger RP was associated with smaller RTs $\left(t_{(17)}=2.51, p<0.05\right)$, while for incongruent trials larger RP was associated with slower responses $\left(t_{(17)}=2.21, p<0.05\right)$.

This finding suggests that increased readiness to make a voluntary action, as reflected in RP, speeds reaction time when the stimulus-driven responses is congruent and slows reaction time when an incongruent response is required. The corresponding analysis for accuracy revealed only a significant main effect of congruency $\left(F_{(1,17)}=31.9, p<0.001\right)$, but no bin by congruency interaction $\left(F_{(1,17)}=2.7\right)$ and no three-way interaction $\left(F_{(1,17)}=\right.$ 1.2). The absence of any effects for the LRP amplitude again highlights that the trials where participants successfully responded to the target were not associated with significant motor lateralization.

Since this final analysis was only conducted on a subset of trials that were determined to have been genuine responses to the stimuli, it is important to rule out the possibility that this filtering did not unduly influence this association between prestimulus motor potentials and reaction times. Therefore, we repeated this analysis using a fixed reaction time cutoff of $100 \mathrm{~ms}$ for both congruent and incongruent responses rather than the individually determined cutoffs used in the previous analysis. The results of this analysis showed a very similar pattern, namely significantly faster responses associated with greater RP for congruent trials $\left(t_{(17)}=2.51, p<0.05\right)$, with the opposite association observed for incongruent trials $\left(t_{(17)}=2.22, p<0.05\right)$. Indeed, the observation in the previous analysis that RP amplitude was independent of the ability of participants to inhibit their voluntary action strongly suggests that this important association between RP amplitude and target RT should be stable regardless of the RT cutoff employed. Similarly, this also likely rules out the possibility that the association between RP and RT on congruent trials could be caused by some voluntary action trials being misclassified as target responses, since these trials would be among the fastest responses but would not have a significantly larger RP amplitude.

\section{Discussion}

The present study investigated the relationship between voluntary and stimulus-driven action control. Our aim was to provide further evidence regarding how these two systems interact and to assess whether central aspects of them might overlap in the brain. To do so, we presented two alternative forced choice stimuli during participants' preparation of a voluntary action with the leftor right-hand. As a consequence, the external stimulus coded for one of two responses that could be either congruent or incongruent with participants' voluntary action preparation. By assessing both RTs to the forced choice stimulus as well as indices of voluntary motor preparation before stimulus presentation, we were able to analyze stimulus-driven behavior as a function of voluntary motor preparation.

In reference to the four possible hypotheses outlined in the introduction, our results are consistent with a central overlap between voluntary and stimulus-driven action preparation. We found that on those trials where participants were able to abort their voluntary action to make the stimulus-driven response, $\mathrm{RP}$ amplitude at the time of stimulus onset predicted how quickly participants would respond to the stimulus. More precisely, for congruent trials increased voluntary action preparation was associated with faster stimulus-driven responses, whereas for incongruent trials large RPs led to slower responses. Since the RP is normally thought of as a marker of the engagement of voluntary action mechanisms (Praamstra et al., 1996; Waszak et al., 2005; Haggard, 2008), this suggests that aspects of voluntary and 
stimulus-driven action preparation overlap in medial frontal cortex.

Our results are inconsistent with the possibility that activation of the intentional action system interferes with stimulus-driven system responding (Astor-Jack and Haggard, 2005), since this would predict a slowing of RTs for increased voluntary action preparation even for congruent trials. Furthermore, this hypothesis should predict that advanced voluntary action preparation should result in reduced accuracy of the stimulus-driven system, whereas we observed no association between prestimulus RP and response accuracy. Note that, over and above the dependency of stimulus-driven RTs on prestimulus RPs described above, we found that responding of participants to external stimuli was impeded by the concurrent preparation of voluntary actions, as shown by increased RTs compared to the choice RT task without concurrent voluntary action preparation. This replicates previous findings that have been interpreted in terms of interference (Obhi and Haggard, 2004; Astor-Jack and Haggard, 2005). However, our data suggest that the comparison of reaction times in blocks containing voluntary actions and those with no voluntary actions may not be indicative of interference between voluntary and stimulus-driven behavior. Rather, this may simply reflect differences in attentional demands between the two tasks, such that participants are able to more efficiently allocate their attention to the need to make a stimulus-driven response when they are not concurrently preparing a voluntary action. Moreover, the negative association between prestimulus negativities and RT for incongruent trials also strongly argues against a contingent negative variation explanation for our data. Rather, we suggest that voluntary action preparation as measured by the RP is partially transferable to stimulus-driven action preparation.

Our findings are consistent with recent experiments conducted by Obhi and colleagues who showed that switching from one voluntary action to a different stimulus-driven action (Obhi et al., 2009a), or to an action with a different effector or a different hand (Obhi et al., 2009b), results in a greater RT cost than switching to the same action. The additional use of EEG in the present experiment allowed us to significantly extend these previous findings by investigating the relationship between the degree of congruent and incongruent voluntary action preparation and the speed of stimulus driven responding. This is important because our behavioral result alone is not sufficient to conclude that voluntary action preparation can be transferred to stimulus-driven reactions, since the slower RT for incongruent trials may reflect the additional conflict involved in switching not only between different action types (voluntary and stimulus driven) but also between different effectors (left- or right-hand actions). Similarly, the increased RT for more advanced incongruent voluntary action preparation might also reflect a greater degree of betweenhand conflict. However, the reduced RT for congruent trials when voluntary action preparation was more advanced strongly suggests that stimulus-driven action selection can be facilitated by voluntary action preparation. This latter result is also consistent with the observation of Obhi et al. (2009b) that if a stimulus appeared in the period immediately before the onset of the planned voluntary action, then the RT cost is significantly reduced. The present study further validates this finding by directly measuring voluntary action preparation and extends it by showing an interaction between the degree of congruent and incongruent voluntary action preparation and the speed of stimulus-driven responding.

Two more aspects of our results deserve further discussion. First, analysis of prestimulus motor components on target trials revealed significant motor lateralization only in trials where participants were unable to overcome their voluntary action to perform the stimulus-driven response. This suggests that when voluntary motor preparation has reached this final stage (i.e., lateralized preparation), it is too late to interrupt its execution. The amplitude of the LRP at the point at which a stop signal is presented has previously been shown to influence participants' ability to inhibit their response to a go signal (Band and van Boxtel, 1999; van Boxtel et al. 2001). However, in the stop signal paradigm participants are typically able to inhibit their response upon presentation of the stop signal despite the presence of a significant (albeit smaller) LRP. The lack of significant LRP on successful target responses in the current experiment may point toward the possibility that lateralized motor activity resulting from voluntary action preparation is more difficult to inhibit than the same level of activity resulting from stimulus-driven response preparation. The apparent absence of ipsilateral inhibition in our lateralized motor potentials, previously observed in choice reaction tasks (e.g., Vidal et al. 2003; Praamstra and Seiss, 2005; Meynier et al., 2009), further suggests that despite using similar brain systems, voluntary and stimulus-driven actions may differ in terms of the precise interaction of different processes at varying stages of action preparation. Obhi et al. (2009b) propose that voluntary action preparation might reflect a balance between general inhibitory processes and a release of inhibition for the planned action. This suggestion is difficult to fully assess in the present study, since activation of different parts of medial frontal cortex reflecting both preparatory and inhibitory processes may contribute toward the activity we measured over central electrodes. Future research should attempt to further explore the role of inhibitory processes in the interaction between voluntary and stimulus-driven actions in more detail to build a better understanding of the precise processes involved in preparing an action based on an internal representation (such as a goal) and on an external stimulus.

Second, it was important for our experiment to be able to determine which voluntary action participants had been preparing before stimulus onset. We achieved this by asking participants, at the end of the trial, to rate their voluntary action preparation. Aside from a small number of participants (who were excluded from further analysis) who were unable to reliably classify their action preparation, the majority of participants appeared to be able to meaningfully rate their voluntary response. Validation of their ratings comes from the significant LRPs for those fast reactions that were deemed to be the continued execution of their voluntary action. The observation that the trials classed as incongruent were responded to more slowly and less accurately suggests that these subjective ratings were also accurate in trials where participants made genuine stimulus-driven responses. The preserved ability to introspect on hand-specific action preparation despite the absence of an LRP for these trials is perhaps somewhat surprising given previous research strongly associating LRP with the conscious intention (Haggard and Eimer, 1999) but is in line with other research associating RP amplitude with the conscious experience of intention (Libet et al., 1983; Lau et al., 2004). In addition, Fried et al. (1991) showed that direct stimulation of neurons in SMA induces limb-specific intention to perform actions, which at a higher voltage results in a movement in the same limb. This somatotopic organization of SMA is consistent with our observation that RP activation is sufficient for allowing participants to introspect on a limb-specific intention. 


\section{Conclusions}

Our data provide evidence that the degree of congruent or incongruent voluntary action preparation at the onset of a target stimulus differentially predicts participants' RT to the upcoming stimulus. This shows that the stimulus-driven system can profit from congruent activation in the voluntary action system, suggesting that the two systems share common central preparatory mechanisms.

\section{References}

Astor-Jack T, Haggard P (2005) Intention and reactivity. In: Attention in action: advances from cognitive neuroscience (Humphreys GW, Riddoch MJ, eds), pp 109-131. New York: Psychology Press.

Band GP, van Boxtel GJ (1999) Inhibitory motor control in stop paradigms: review and reinterpretation of neural mechanisms. Acta Psychologica 101:179-211.

Boccardi E, Della Sala S, Motto C, Spinnler H (2002) Utilisation behaviour consequent to bilateral SMA softening. Cortex 38:289-308.

Coles MG (1989) Modern mind-brain reading: psychophysiology, physiology, and cognition. Psychophysiology 26:251-269.

Deiber MP, Honda M, Ibañez V, Sadato N, Hallett M (1999) Mesial motor areas in self-initiated versus externally triggered movements examined with fMRI: effect of movement type and rate. J Neurophysiol 81:3065-3077.

Delorme A, Makeig S (2004) EEGLAB: an open source toolbox for analysis of single-trial EEG dynamics including independent component analysis. J Neurosci Methods 134:9-21.

Fried I, Katz A, McCarthy G, Sass KJ, Williamson P, Spencer SS, Spencer DD (1991) Functional organization of human supplementary motor cortex studied by electrical stimulation. J Neurosci 11:3656-3666.

Haggard P (2008) Human volition: towards a neuroscience of will. Nat Rev Neurosci 9:934-946.

Haggard P, Eimer M (1999) On the relation between brain potentials and the awareness of voluntary movements. Exp Brain Res 126:128-133.

Hughes G, Waszak F (2011) ERP correlates of action effect prediction and visual sensory attenuation in voluntary action. Neuroimage $56: 1632-1640$.

Jenkins IH, Jahanshahi M, Jueptner M, Passingham RE, Brooks DJ (2000) Self-initiated versus externally triggered movements. II. The effect of movement predictability on regional cerebral blood flow. Brain 123:1216-1228.

Krieghoff V, Waszak F, Prinz W, Brass M (2011) Neural and behavioral correlates of intentional actions. Neuropsychologia 49:767-776.

Kurata K, Wise SP (1988) Premotor and supplementary motor cortex in rhesus monkeys: neuronal activity during externally- and internallyinstructed motor tasks. Exp Brain Res 72:237-248.

Lau HC, Rogers RD, Haggard P, Passingham RE (2004) Attention to intention. Science 303:1208-1210

Libet B, Gleason CA, Wright EW, Pearl DK (1983) Time of conscious intention to act in relation to onset of cerebral activity (readiness-potential). The unconscious initiation of a freely voluntary act. Brain 106:623-642.

Meynier C, Burle B, Possamaï CA, Vidal F, Hasbroucq T (2009) Neural inhibition and interhemispheric connections in two-choice reaction time: a Laplacian ERP study. Psychophysiology 46:726-730.

Nachev P, Kennard C, Husain M (2008) Functional role of the supplementary and pre-supplementary motor areas. Nat Rev Neurosci 9:856-869.

Obhi SS, Haggard P (2004) Internally generated and externally triggered actions are physically distinct and independently controlled. Exp Brain Res 156:518-523.

Obhi SS, Matkovich S, Gilbert SJ (2009a) Modification of planned actions. Exp Brain Res 192:265-274.

Obhi SS, Matkovich S, Chen R (2009b) Changing the "when" and "what" of intended actions. J Neurophysiol 102:2755-2762.

Passingham RE, Bengtsson SL, Lau HC (2010) Medial frontal cortex: from self-generated action to reflection on one's own performance. Trends Cogn Sci 14:16-21.

Praamstra P, Seiss E (2005) The neurophysiology of response competition: motor cortex activation and inhibition following subliminal response priming. J Cogn Neurosci 17:483-493.

Praamstra P, Stegeman DF, Horstink MW, Cools AR (1996) Dipole source analysis suggests selective modulation of the supplementary motor area contribution to the readiness potential. Electroencephalogr Clin Neurophysiol 98:468-477.

Romo R, Schultz W (1987) Neuronal activity preceding self-initiated or externally timed arm movements in area 6 of monkey cortex. Exp Brain Res 67:656-662.

van Boxtel GJ, van der Molen MW, Jennings JR, Brunia CH (2001) A psychophysiological analysis of inhibitory motor control in the stop-signal paradigm. Biol Psychol 58:229-262.

Vidal F, Grapperon J, Bonnet M, Hasbroucq T (2003) The nature of unilateral motor commands in between-hand choice tasks as revealed by surface Laplacian estimation. Psychophysiology 40:796-805.

Walter WG, Winter AL, Cooper R, Mccallum WC, Aldridge VJ (1964) Contingent negative variation: an electric sign of sensorimotor association and expectancy in human brain. Nature 203:380-384.

Waszak F, Wascher E, Keller P, Koch I, Aschersleben G, Rosenbaum DA, Prinz W (2005) Intention-based and stimulus-based mechanisms in action selection. Exp Brain Res 162:346-356. 